\title{
Palladium Nanoparticle-Graphene Hybrids as Active Catalysts for the Suzuki Reaction
}

\author{
Yang Li, Xiaobin Fan $(\varangle)$, Junjie Qi, Junyi Ji, Shulan Wang, Guoliang Zhang, and Fengbao Zhang \\ School of Chemical Engineering and Technology, Tianjin University, Tianjin 300072, China \\ Received: 18 January 2010 / Revised: 12 April 2010 / Accepted: 22 April 2010 \\ (C) The Author(s) 2010. This article is published with open access at Springerlink.com
}

\begin{abstract}
Graphene has been successfully modified with palladium nanoparticles in a facile manner by reducing palladium acetate $\left[\mathrm{Pd}(\mathrm{OAc})_{2}\right]$ in the present of sodium dodecyl sulfate (SDS), which is used as both surfactant and the reducing agent. The palladium nanoparticle-graphene hybrids ( $\mathrm{Pd}$-graphene hybrids) are characterized by highresolution transmission electron microscopy, atomic force microscopy, Raman spectroscopy, X-ray photoelectron spectroscopy, X-ray diffraction, and energy dispersive X-ray spectroscopy. We demonstrate that the Pd-graphene hybrids can act as an efficient catalyst for the Suzuki reaction under aqueous and aerobic conditions, with the reaction reaching completion in as little as $5 \mathrm{~min}$. The influence of the preparation conditions on the catalytic activities of the hybrids is also investigated.
\end{abstract}

\section{KEYWORDS}

Graphene, Pd nanoparticles, hybrids, catalyst, Suzuki reaction

\section{Introduction}

Graphene is the name given to a two-dimensional sheet of $\mathrm{sp}^{2}$-hybridized carbon. Due to its extraordinary thermal, mechanical, and electrical properties, graphene has aroused an explosion of interest both for theoretical studies and applications [1-6]. Graphene with a high specific surface area [7] has great potential in the development of new kinds of composite materials [8], especially as a substrate to host metal nanoparticles [8-11]. However, only a few studies have involved the application of graphene-based materials as heterogeneous catalysts [12-14].

In the field of catalysis, palladium appears to be particularly important among the noble metals [15]. As an efficient catalyst in organic reactions, it can offer the most favorable combination of activity and selectivity [16]. One of the well-known reactions catalyzed by palladium is the Suzuki coupling reaction, which is a powerful and convenient synthetic method in organic chemistry to generate biaryls, conducting polymers, and liquid crystals [17-21].

We have previously found that graphene oxide can undergo a deoxygenation reaction under alkaline condition, providing a nontoxic, economical, and scalable way to prepare graphene [22]. Here we show that palladium nanoparticles can be homogeneously dispersed on chemically modified graphene by a facile route. The resulting palladium nanoparticle-graphene hybrids (Pd-graphene hybrids) with controllable size of palladium nanoparticles were shown to act as efficient catalysts for the Suzuki reaction in aqueous systems.

Address correspondence to xiaobinfan@tju.edu.cn 


\section{Experimental}

\subsection{Synthesis of Pd-graphene hybrids}

Graphite oxide was prepared by the Hummers method [23] and exfoliated into graphene oxide (GO) by sonication in water. The resulting GO was heated in sodium hydroxide solution to obtain a pristine graphene suspension [22]. Then, $20 \mathrm{~mL}$ of sodium dodecyl sulfate (SDS, $0.1 \mathrm{~mol} / \mathrm{L}$ ) aqueous solution was added to $10 \mathrm{~mL}$ of the graphene suspension $(\sim 2.5 \mathrm{mg} / \mathrm{mL})$ and sonicated for $5 \mathrm{~min}$. Subsequently, $10 \mathrm{mg}$ of palladium acetate $\left(\mathrm{Pd}(\mathrm{OAc})_{2}\right)$ was added, and then the mixture was refluxed at $110{ }^{\circ} \mathrm{C}$ with magnetic stirring for $4 \mathrm{~h}$. After cooling to room temperature, the mixture was then washed extensively with deionized water and centrifuged several times, in order to remove excess surfactant.

\subsection{Suzuki reaction catalyzed by Pd-graphene hybrids}

Iodobenzene ( $204 \mathrm{mg}, 1.0 \mathrm{mmol}$ ) was added to a stirred mixture of SDS (144 mg, $0.5 \mathrm{mmol}$ ), tripotassium phosphate $\left(\mathrm{K}_{3} \mathrm{PO}_{4}, 399 \mathrm{mg}\right)$, phenylboronic acid (146 mg, $1.2 \mathrm{mmol})$, and deionized water $\left(\mathrm{H}_{2} \mathrm{O}\right)(20 \mathrm{~mL})$, followed by $\mathrm{Pd}$-graphene hybrids ( $1.1 \mathrm{~mol} \%)$. The mixture was then stirred at $100{ }^{\circ} \mathrm{C}$ in oil bath for only $5 \mathrm{~min}$ (Scheme 1) and then extracted with ethyl acetate $(3 \times 20 \mathrm{~mL})$. The combined organic extract was dried over anhydrous sodium sulfate $\left(\mathrm{Na}_{2} \mathrm{SO}_{4}\right)$, and the resulting mixture was analyzed by gas chromatography (GC). The catalysts were recovered by simple centrifugation and washed extensively with deionized water.

\subsection{Characterization}

The Pd-graphene hybrids were characterized by high-resolution transmission electron microscopy (HRTEM) (Philips Tecnai G2 F20), energy dispersive X-ray spectroscopy (EDX) (Philips Tecnai G2 F20), atomic force microscopy (AFM) (CSPM 5000), X-ray photoelectron spectroscopy (XPS) (PerkinElmer, PHI 1600 spectrometer), and Raman spectroscopy (NT-MDT NTEGRA Spectra). The X-ray diffraction (XRD) was performed on a Bruker-Nonius D8 FOCUS diffractometer. The catalytic activity of $\mathrm{Pd}$-graphene hybrids was measured by GC, which was performed on an Agilent 6890N GC-FID system.

\section{Results and discussion}

\subsection{Synthesis of Pd-graphene hybrids}

Since most oxidized functional groups are removed during the reduction of graphene oxide, it is generally difficult to disperse the resulting graphene in solution. SDS is used as the surfactant and reducing agent in the synthesis of the Pd-graphene hybrids, since on heating it decomposes to 1-dodecanol which is subsequently oxidized to dodecanoic acid when it reduces the $\mathrm{Pd}(\mathrm{II})$ precursor to $\mathrm{Pd}$ [24]. As a result, the Pd-graphene hybrids have excellent dispersability, and no precipitation was observed after several months (Fig. S-1 in the Electronic Supplementary Material (ESM)). The presence of dodecanoic acid and unreacted SDS not only facilitates the dispersability of the resulting hybrids, but also contributes to the homogeneous distribution of $\mathrm{Pd}$ nanoparticles on the graphene sheets. The alkyl chains of residual SDS molecules lie on the surface of the graphene sheets, with the sulfonate groups (Fig. S-2 in the ESM) extending into the solution to provide electrostatic repulsion that stabilizes the suspension. These sulfonate groups also behave as both the active sites for the adsorption of $\mathrm{Pd}$ ions and the nucleation centers for Pd nanoparticles [25].

In this way, Pd nanoparticles are grown and densely assembled on the graphene surface, as shown in Figs. 1(a) and 1(b). A statistical analysis of the size distribution of the Pd nanoparticles seen in HRTEM, obtained from different regions of the samples, was performed. The mean size of the $\mathrm{Pd}$ nanoparticles dispersed on the graphene sheets is about $4 \mathrm{~nm}$. The presence of a large amount of $\mathrm{Pd}$ nanoparticles on graphene is confirmed by EDX analysis (Fig. 1(c)). The oxygen and sulfur signals in Fig. 1(c) arise from the residual dodecanoate and sulfonate groups.

The uniform decoration of $\mathrm{Pd}$ nanoparticles on the surface of graphene is supported by AFM images of the Pd-graphene on a mica substrate (Fig. 2). Comparison with the AFM image of the graphene suspension (Fig. S-3 in the ESM) indicates that the 

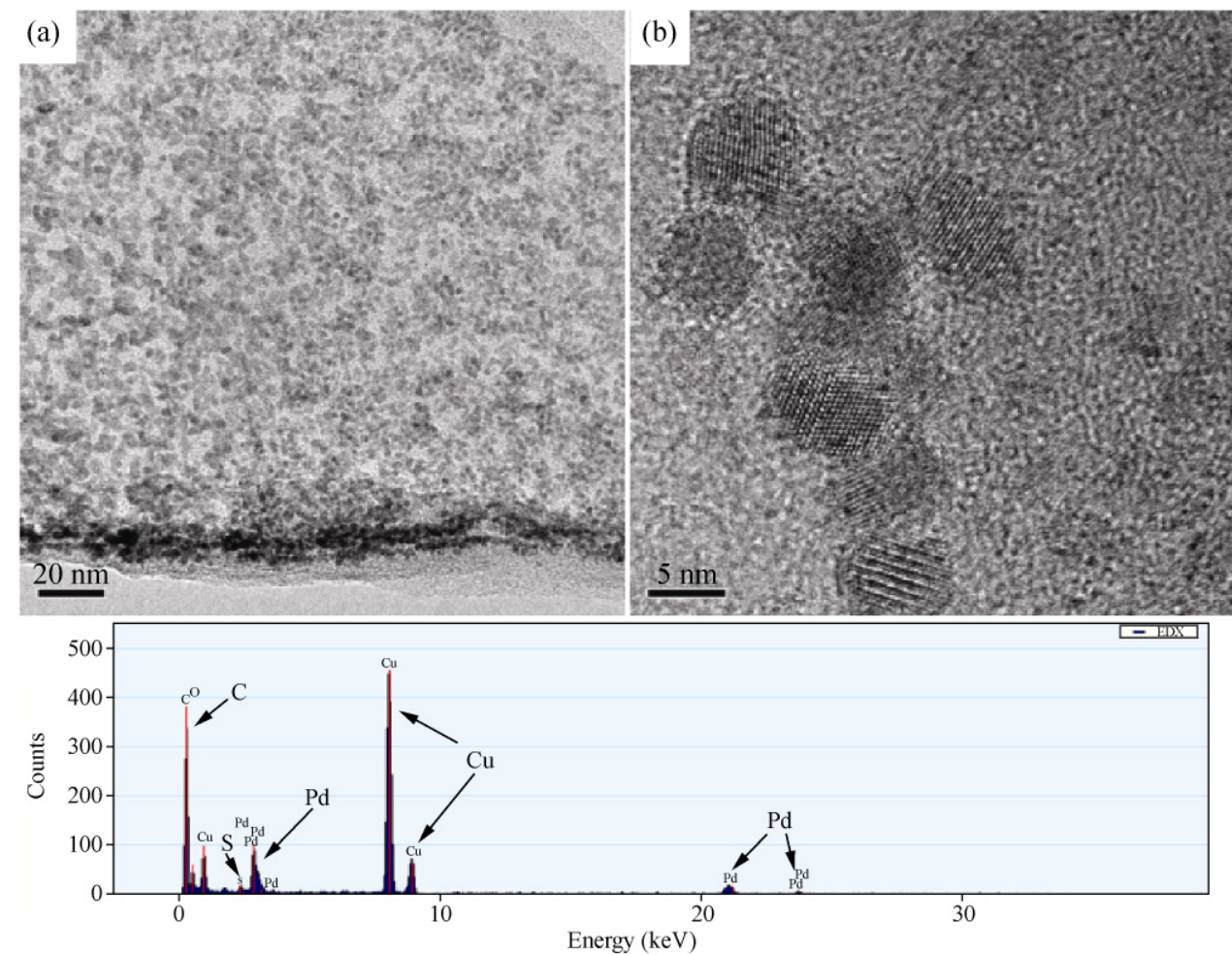

(c)

Figure 1 (a) TEM image and (b) corresponding HRTEM image of Pd nanoparticles homogeneously decorated on graphene. (c) EDX result for $\mathrm{Pd}$-graphene hybrids (Note that the $\mathrm{Cu}$ peaks come from the copper grid.)

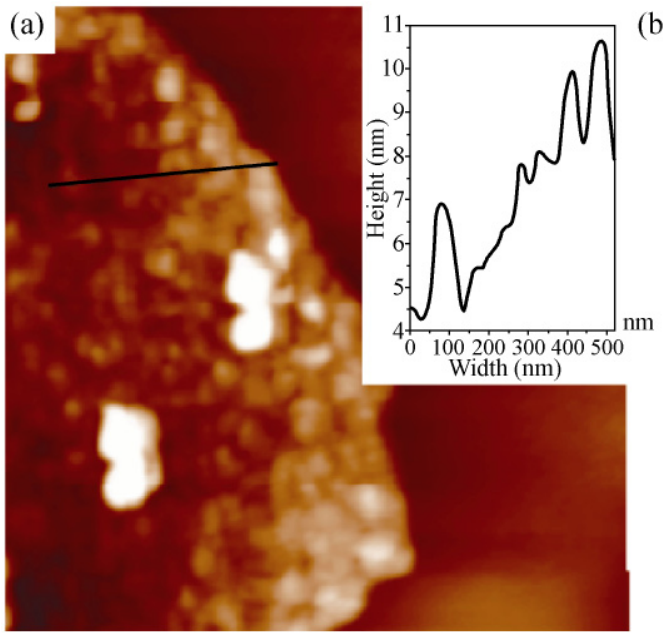

(b)

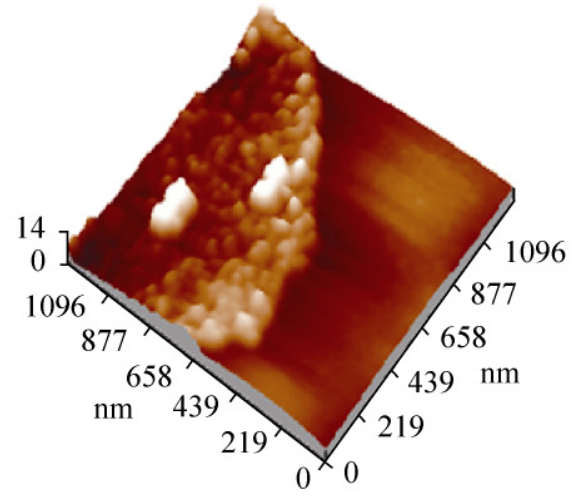

Figure 2 (a) AFM image of Pd-graphene hybrids deposited on a freshly cleaved mica substrate, where the bright "spots" are the Pd nanoparticles deposited on the graphene and the insert image shows a cross-section analysis, and (b) the corresponding 3-D image of the Pd-graphene hybrid 
height of the $\mathrm{Pd}$ nanoparticles on graphene is in the range $2-14 \mathrm{~nm}$, consistent with the crystallite size calculated from the XRD pattern (Fig. S-4 in the ESM). Note that the lateral dimensions of the nanoparticles are broadened by the tip dilation of AFM [26]. It should be noted that as there are no nanoparticles outside the graphene sheets, the nanoparticles seem to be tightly bound on the surface of graphene, even after intensive sonication during the preparation of the sample for AFM characterization. Considering that the interatomic distance in $\mathrm{Pd}(0.225 \mathrm{~nm})$ is close to the honeycomb spacing in graphene $(0.246 \mathrm{~nm})$, the strong interaction of the Pd nanoparticles and graphene may also be explained as epitaxial absorption [27].

Raman spectra of GO, graphene, and $\mathrm{Pd}$-graphene hybrids are shown in Fig. 3. The two bands at about $1358 \mathrm{~cm}^{-1}$ and $1622 \mathrm{~cm}^{-1}$ correspond to the disorderinduced D band and the in-phase vibration of the graphene lattice ( $\mathrm{G}$ band). Compared with that for GO (Fig. 3(a)), the $I(\mathrm{D}) / I(\mathrm{G})$ intensity ratio for graphene in Fig. 3(b) is slightly higher, which may indicate that the graphene sheets have become smaller in size [28]. The $I(\mathrm{D}) / I(\mathrm{G})$ intensity ratio of Pd-graphene (Fig. 3(c)) is higher than that of graphene. Such an enhancement has also been observed in examples of GO [29] and carbon nanotubes $[30,31]$ decorated with metal nanoparticles, indicating a probable chemical interaction or bond between the metal nanoparticles and graphene $[29,30]$. As the graphene in this study is obtained by chemical reduction of GO, there must be many carbon vacancies and defects [12], which may enhance the interaction between Pd nanoparticles and graphene [32, 33]. Moreover, the $G$ bands $\left(1622 \mathrm{~cm}^{-1}\right)$ of graphene and $\mathrm{Pd}$-graphene hybrids are shifted to lower frequency compared with that of GO $\left(1629 \mathrm{~cm}^{-1}\right)$, which can probably be explained by damage to the alternating pattern of single and double carbon bonds within $\mathrm{sp}^{2}$ carbon ribbons during the reaction [34].

Notably, the size distribution of $\mathrm{Pd}$ nanoparticles decorated on graphene sheets can be controlled by varying the concentration of SDS used in the reaction. As shown in Fig. 4(a), homogenous and condensed decoration of graphene with Pd nanoparticles of about $4 \mathrm{~nm}$ in size can be readily achieved by using SDS with a concentration of $0.1 \mathrm{~mol} / \mathrm{L}$ (Figs. $4(\mathrm{a})$ and $4(\mathrm{~b})$ ). In contrast, a lower concentration of SDS $(0.05 \mathrm{~mol} / \mathrm{L})$ results in a scattering of $\mathrm{Pd}$ nanoparticles with a mean size of about $15 \mathrm{~nm}$ (Fig. 4(c)). These results can be explained by the rapid formation of more embryonic $\mathrm{Pd}$ nanoparticles in the presence of more surfactant (SDS) $[35,36]$.

The different sizes of the Pd nanoparticles formed on graphene were also corrobrated by XPS. As shown in XPS spectra (Fig. 5), the binding energy $\left(3 d_{5 / 2}\right)$ of the smaller Pd nanoparticles $(\sim 4 \mathrm{~nm})$ decorated on graphene (Fig. 5(a)) shows an obvious shift of $0.46 \mathrm{eV}$

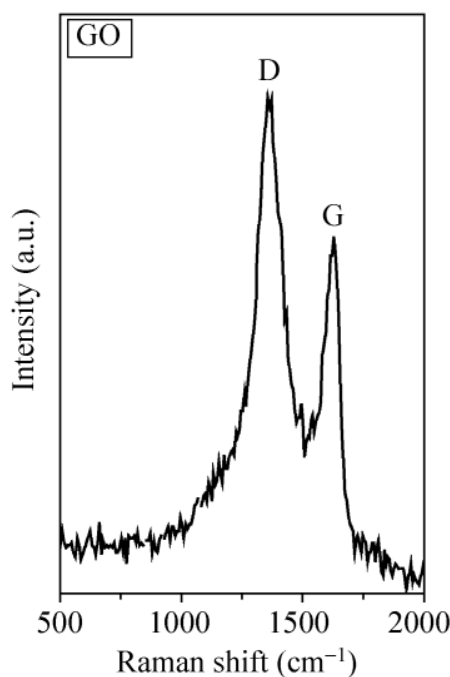

(a)

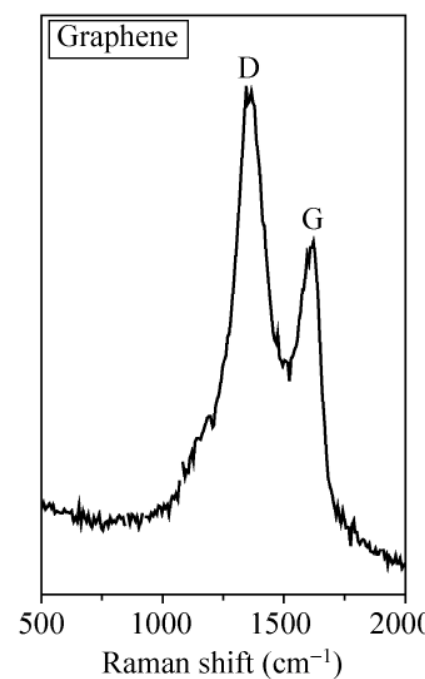

(b)

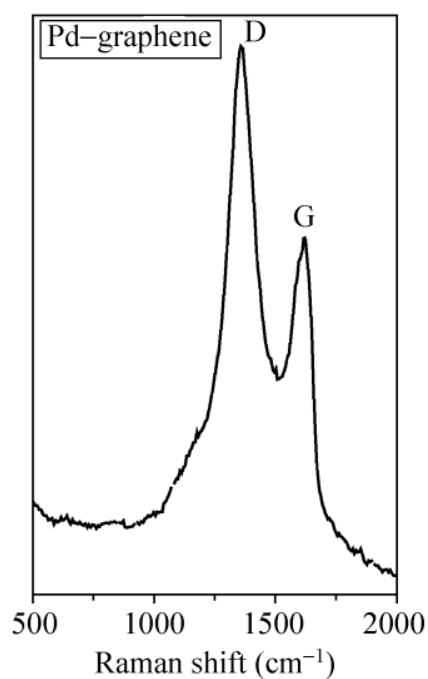

(c)

Figure 3 Raman spectra of (a) graphene oxide, (b) graphene, and (c) Pd-graphene hybrid 

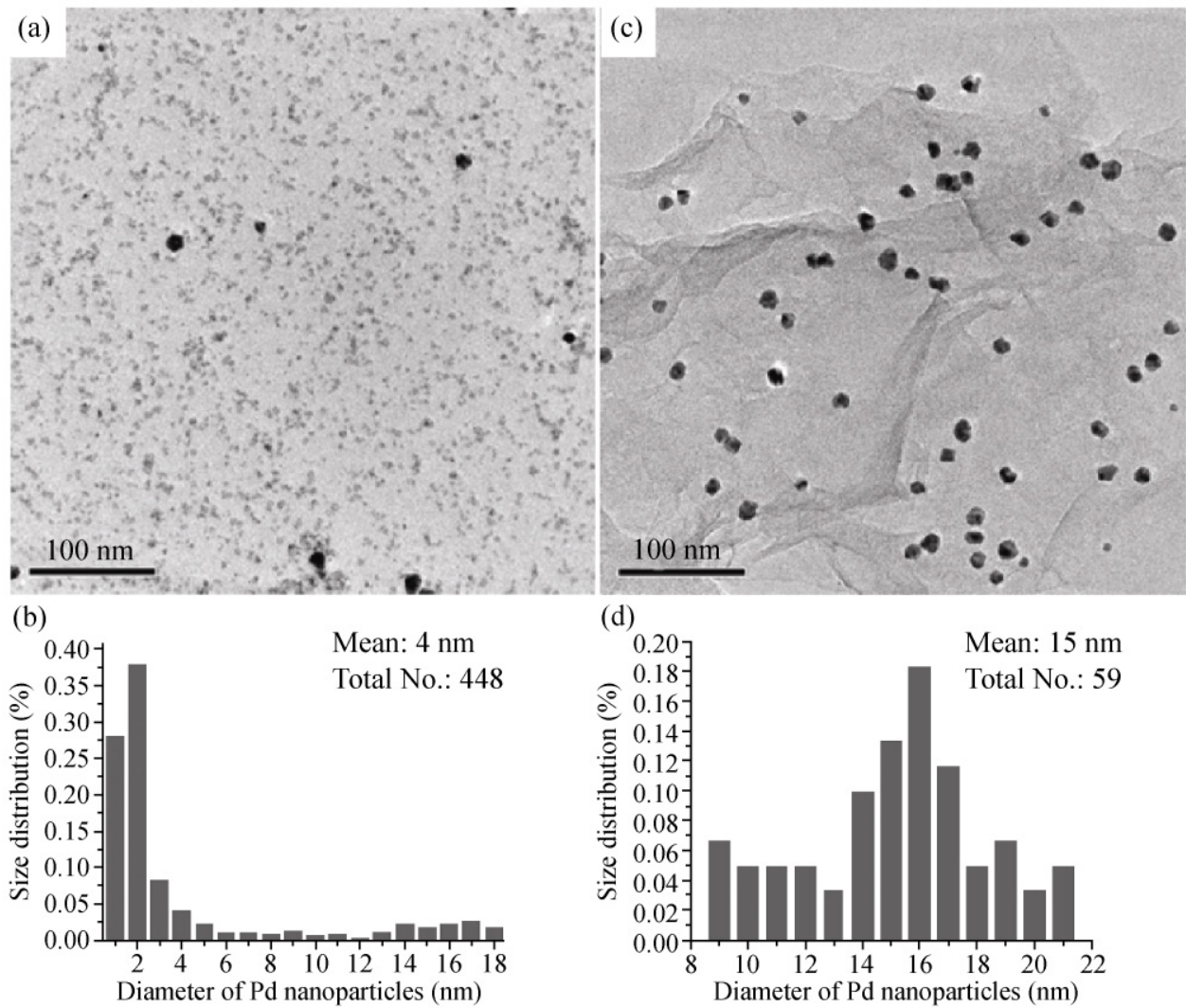

Figure 4 (a) HRTEM image and (b) size distribution of Pd nanoparticles on graphene prepared using 0.1 mol/L SDS. (c) HRTEM image and (d) size distribution of Pd nanoparticles on graphene prepared using $0.05 \mathrm{~mol} / \mathrm{L} \mathrm{SDS}$

towards high binding energy compared with the value for the larger Pd nanoparticles ( 15 nm) (Fig. 5(b)). Such shifts in binding energy with particle size have been observed for metal nanoparticles on a variety of supports, and are generally attributed to the effect of the average coordination number or cluster size on the core level binding energy [37]. The XPS spectra, together with valence band spectra of these two samples (Fig. S-5 in the ESM), strongly suggest that the electronic structure varies with the size of the metal particles [38].

\subsection{Suzuki reaction catalyzed by Pd-graphene hybrids}

The catalytic activity of the Pd-graphene hybrids in the formation of biaryl carbon-carbon bonds was investigated using the Suzuki reaction of iodobenzene with phenylboronic acid (as shown in Scheme 1). Normally, Suzuki reactions are carried out in a mixture of an organic solvent and an aqueous inorganic base, generally under an inert atmosphere $[21,39,40]$. When the reaction is performed in aqueous systems under aerobic conditions, activation by phosphine ligands is usually required [41]. In this study, however, we investigate the $\mathrm{Pd}$-graphene catalyzed Suzuki reaction in water containing SDS and $\mathrm{K}_{3} \mathrm{PO}_{4}$ under aerobic conditions, without any pre-activation. (The GC results are shown in Fig. S-6 in the ESM.)

To optimize the reaction conditions, a series of experiments with different reaction temperatures and quantities of Pd-graphene catalysts were carried out with $4 \mathrm{~nm}$ Pd nanoparticles as illustrated in Fig. S-7 in the ESM. It was found that the best result in terms of

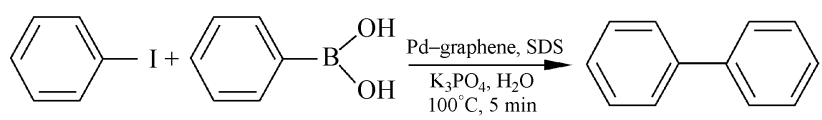

Scheme 1 Suzuki reaction 


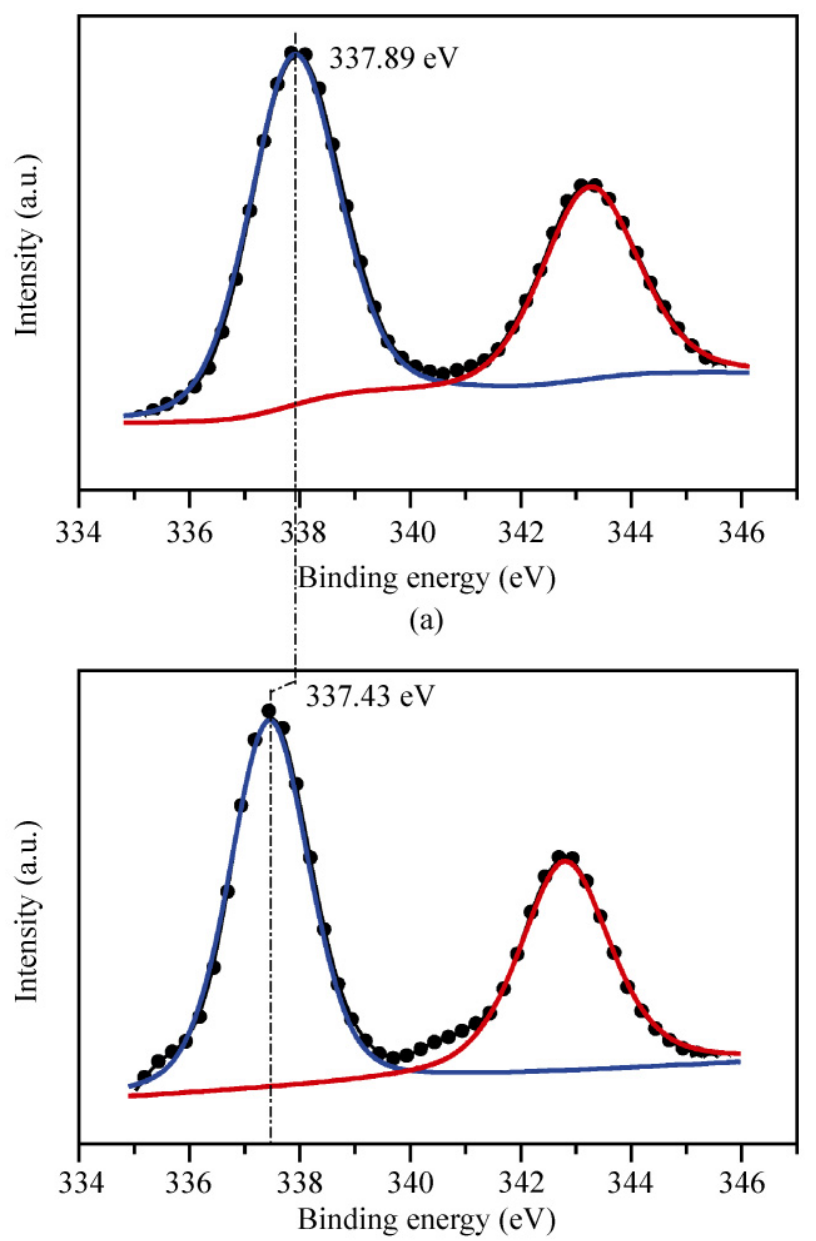

(b)

Figure 5 (a) Pd $3 d$ electron region of the X-ray photoelectron spectrum of Pd-graphene hybrids prepared using $0.1 \mathrm{~mol} / \mathrm{L}$ SDS (the average diameter of Pd nanoparticles is $\sim 4 \mathrm{~nm}$ ) and (b) the corresponding spectrum of large Pd nanoparticles $(\sim 15 \mathrm{~nm})$ on graphene prepared using $0.05 \mathrm{~mol} / \mathrm{L} \mathrm{SDS}$

yield was obtained by using $1.1 \mathrm{~mol} \%$ Pd-graphene catalyst at $100{ }^{\circ} \mathrm{C}$, when the yield and selectivity reached as high as $100 \%$ and $95.5 \%$, respectively. When carried out without SDS, the yield was only $75.7 \%$. Although the presence of SDS leads to a marked improvement in the yield of the Suzuki reaction, it does complicate the reaction to some extent [42].

The reactions are were also carried out under similar conditions using Pd nanoparticles synthesized in the presence of SDS without graphene (the synthesis is described in Scheme S-1, in the ESM), and $\mathrm{Pd}(\mathrm{OAc})_{2}$. The yields were $86 \%$ and $44.3 \%$, respectively, much lower than when using equivalent amounts of $\mathrm{Pd}-$ graphene catalysts. The excellent catalytic activity of Pd-graphene catalysts may result from the graphene sheets stabilizing the $\mathrm{Pd}$ nanoparticles against aggregation.

Table 1 summarizes the performance of the different $\mathrm{Pd}$-graphene catalysts under the optimum reaction conditions. The results confirm that $4 \mathrm{~nm}$ Pd nanoparticles supported on graphene (prepared with $0.1 \mathrm{~mol} / \mathrm{L} \mathrm{SDS}$ ) are more catalytically active than $15 \mathrm{~nm}$ $\mathrm{Pd}$ nanoparticles (prepared with $0.05 \mathrm{~mol} / \mathrm{L}$ SDS). The better catalytic activity of the smaller nanoparticles decorated on graphene can be attributed to the larger number of active sites on the smaller Pd nanoparticles [43]. The size-dependent variation in electronic structure may also contribute to the better catalytic properties of the smaller Pd nanoparticles [38]. This result is consistent with other reports that the activity of $\mathrm{Pd}$ catalysts in $\mathrm{C}-\mathrm{C}$ bond forming reactions, such as the Suzuki reaction, varies markedly with particle size [44, 45], and the ability to precisely control the size of the Pd nanoparticles formed on graphene is therefore a significant advantage of our method.

The Pd-graphene catalyst can also be reused successfully. The results in Table 2 show that the $\mathrm{Pd}$-graphene hybrid retained a reasonable performance when reused in ten cycles. (The HRTEM images and EDX analysis of the Pd-graphene hybrids after ten cycles are shown in Fig. S-8 in the ESM.)

As a comparison, the dispersion and catalytic activity of $\mathrm{Pd}$-graphene hybrids after reaction in the absence

Table 1 Catalytic results for different Pd-graphene hybrids under same reaction conditions

\begin{tabular}{ccc}
\hline Catalyst $^{\mathrm{a}}$ & Yield (\%) & Selectivity (\%) \\
\hline $\begin{array}{c}\text { Pd-graphene with } 4 \mathrm{~nm} \mathrm{Pd} \\
\text { nanoparticles }\end{array}$ & 100 & 95.5 \\
$\begin{array}{c}\text { Pd-graphene with } 15 \mathrm{~nm} \mathrm{Pd} \\
\text { nanoparticles }\end{array}$ & 93.7 & 95.2 \\
\hline
\end{tabular}

${ }^{\mathrm{a}}$ Iodobenzene (1.0 mmol), phenylboronic acid (1.2 mmol), SDS $(0.5 \mathrm{mmol}), \mathrm{K}_{3} \mathrm{PO}_{4}(399 \mathrm{mg}), \mathrm{H}_{2} \mathrm{O}(20 \mathrm{~mL})$, and $\mathrm{Pd}$-graphene hybrids $(1.1 \mathrm{~mol} \%) .100{ }^{\circ} \mathrm{C}, 5 \mathrm{~min}$

Table 2 Yields in the Suzuki reaction when the Pd-graphene ${ }^{\mathrm{a}}$ catalyst is reused at $100{ }^{\circ} \mathrm{C}, 5 \mathrm{~min}$

Cycle No. 1st 2nd 3rd 4th 5th 6th 7th 8th 9th 10th

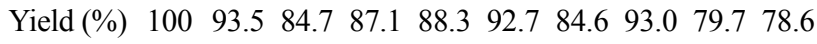

${ }^{\mathrm{a}}$ The average diameter of Pd nanoparticles was $\sim 4 \mathrm{~nm}$ 
of SDS were investigated and the results are illustrated in Fig. S-9 and Table S-1 in the ESM. It was more difficult to separate the $\mathrm{Pd}$-graphene catalysts from the solution, as there is no surfactant participating in the reaction. In addition, the yield of the reaction without SDS decreased to $15 \%$ after 6 cycles (as shown in Table S-1 in the ESM), which may be due to the thick layers of organic reactants/products covering the surface of the hybrids and aggregation of the Pd nanoparticles. This confirms that the presence of SDS has a beneficial effect on the Suzuki reaction.

Bromobenzene and allyl iodide were also employed in the Suzuki reaction with phenylboronic acid to produce the corresponding biaryl and allyl-aryl species. The results are summarized in Table 3. The reactivities are lower than when using iodobenzene as the reactant, which can be attributed to the different strengths of the $\mathrm{C}-\mathrm{I}$ and $\mathrm{C}-\mathrm{Br}$ bonds, as well as the different electronwithdrawing abilities of the halogen substituents [46].

\section{Conclusions}

A simple process has been developed to homogeneously deposit Pd nanoparticles with controllable size distribution on graphene sheets. The resulting Pd-graphene hybrids showed excellent catalytic activity in the Suzuki reaction carried out in water under aerobic condition for a short time, acting as an efficient and easily recovered catalyst for the low-cost and environmentally-friendly synthesis of biaryls. As a low-cost substitute for carbon nanotubes as a support, graphene offers new opportunities for heterogeneous

Table 3 Suzuki reaction of allyl iodide and bromobenzene with phenylboronic acid

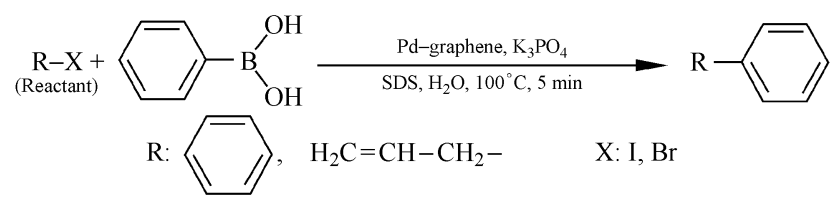

\begin{tabular}{ccc}
\hline Reactant $^{\mathrm{a}}$ & Yield (\%) & Selectivity (\%) \\
\hline Bromobenzene & 29.5 & 89.8 \\
Allyl iodide & 6.5 & 17.2 \\
\hline
\end{tabular}

\footnotetext{
${ }^{\mathrm{a}}$ Reactant $(1.0 \mathrm{mmol})$, phenylboronic acid $(1.2 \mathrm{mmol}), \mathrm{SDS}$ (0.5 mmol), $\mathrm{K}_{3} \mathrm{PO}_{4}(399 \mathrm{mg}), \mathrm{H}_{2} \mathrm{O}(20 \mathrm{~mL})$, and $\mathrm{Pd}$-graphene hybrids ( $\sim \mathrm{nm} \mathrm{Pd}$ nanoparticles, $1.1 \mathrm{~mol} \%$ ). $100{ }^{\circ} \mathrm{C}, 5 \mathrm{~min}$
}

catalysis with metal nanoparticles and this will accelerate the development of applications of graphene.

\section{Acknowledgements}

The authors would like to acknowledge the support of this work by the National Natural Science Foundation of China (20776095) and the Programme of Introducing Talents of Discipline to Universities (No. B06006).

Electronic Supplementary Material: Supplementary material (XPS and XRD results of Pd-graphene hybrids, AFM image of graphene suspension, valence band spectra of different sizes of $\mathrm{Pd}$ nanoparticles on graphene, GC results before and after Suzuki reaction, as well as optimization of the reaction conditions) is available in the online version of this article at http://dx.doi.org/10.1007/s12274-010-0002-z and is accessible free of charge.

Open Access: This article is distributed under the terms of the Creative Commons Attribution Noncommercial License which permits any noncommercial use, distribution, and reproduction in any medium, provided the original author(s) and source are credited.

\section{References}

[1] Stankovich, S.; Dikin, D. A.; Dommett, G. H. B.; Kohlhaas, K. M.; Zimney, E. J.; Stach, E. A.; Piner, R. D.; Nguyen, S. T.; Ruoff, R. S. Graphene-based composite materials. Nature 2006, 442, 282-286.

[2] Ramanathan, T.; Abdala, A. A.; Stankovich, S.; Dikin, D. A.; Herrera-Alonso, M.; Piner, R. D.; Adamson, D. H.; Schniepp, H. C.; Chen, X.; Ruoff, R. S.; Nguyen, S. T.; Aksay, I. A.; Prud'homme, R. K.; Brinson, L. C. Functionalized graphene sheets for polymer nanocomposites. Nat. Nanotechnol. 2008, 3, 327-331.

[3] Meyer, J. C.; Geim, A. K.; Katsnelson, M. I.; Novoselov, K. S.; Booth, T. J.; Roth, S. The structure of suspended graphene sheets. Nature 2007, 446, 60-63.

[4] Ponomarenko, L. A.; Schedin, F.; Katsnelson, M. I.; Yang, R.; Hill, E. W.; Novoselov, K. S.; Geim, A. K. Chaotic Dirac billiard in graphene quantum dots. Science 2008, 320, 356-358.

[5] Novoselov, K. S.; Jiang, Z.; Zhang, Y.; Morozov, S. V.; Stormer, H. L.; Zeitler, U.; Maan, J. C.; Boebinger, G. S.; Kim, P.; Geim, A. K. Room-temperature quantum Hall effect 
in graphene. Science 2007, 315, 1379.

[6] Novoselov, K. S.; Geim, A. K.; Morozov, S. V.; Jiang, D.; Zhang, Y.; Dubonos, S. V.; Grigorieva, I. V.; Firsov, A. A. Electric field effect in atomically thin carbon films. Science 2004, 306, 666-669.

[7] McAllister, M. J.; Li, J. L.; Adamson, D. H.; Schniepp, H. C.; Abdala, A. A.; Liu, J.; Herrera-Alonso, M.; Milius, D. L.; Car, R.; Prud'homme, R. K.; Akasy, I. A. Single sheet functionalized graphene by oxidation and thermal expansion of graphite. Chem. Mater. 2007, 19, 4396-4404.

[8] Hassan, H. M. A.; Abdelsayed, V.; Khder, A. E. R. S.; AbouZeid, K. M.; Terner, J.; El-Shall, M. S.; Al-Resayes, S. I.; El-Azhary, A. A. Microwave synthesis of graphene sheets supporting metal nanocrystals in aqueous and organic media. J. Mater. Chem. 2009, 19, 3832-3837.

[9] Muszynski, R.; Seger, B.; Kamat, P. V. Decorating graphene sheets with gold nanoparticles. J. Phys. Chem. C 2008, 112, 5263-5266.

[10] Stoller, M. D.; Park, S. J.; Zhu, Y. W.; An, J. H.; Ruoff, R. S. Graphene-based ultracapacitors. Nano Lett. 2008, 8, 3498-3502.

[11] Zhou, X. Z.; Huang, X.; Qi, X. Y.; Wu, S. X.; Xue, C.; Boey, F. Y. C.; Yan, Q. Y.; Chen, P.; Zhang, H. In situ synthesis of metal nanoparticles on single-layer graphene oxide and reduced graphene oxide surfaces. J. Phys. Chem. C 2009, 113, 10842-10846.

[12] Yoo, E.; Okata, T.; Akita, T.; Kohyama, M.; Nakamura, J.; Honma, I. Enhanced electrocatalytic activity of Pt subnanoclusters on graphene nanosheet surface. Nano Lett. 2009, 9, 2255-2259.

[13] Scheuermann, G. M.; Rumi, L.; Steurer, P.; Bannwarth, W.; Mulhaupt, R. Palladium nanoparticles on graphite oxide and its functionalized graphene derivatives as highly active catalysts for the Suzuki-Miyaura coupling reaction. J. Am. Chem. Soc. 2009, 131, 8262-8270.

[14] Mastalir, A.; Kiraly, Z.; Patzko, A.; Dekany, I.; L’Argentiere, P. Synthesis and catalytic application of Pd nanoparticles in graphite oxide. Carbon 2008, 46, 1631-1637.

[15] Javey, A.; Guo, J.; Wang, Q.; Lundstrom, M.; Dai, H. J. Ballistic carbon nanotube field-effect transistors. Nature 2003, 424, 654-657.

[16] Blaser, H. U.; Indolese, A.; Schnyder, A.; Steiner, H.; Studer, M. Supported palladium catalysts for fine chemicals synthesis. J. Mol. Catal. A-Chem. 2001, 173, 3-18.

[17] Suzuki, A. Recent advances in the cross-coupling reactions of organoboron derivatives with organic electrophiles, 1995-1998. J. Organomet. Chem. 1999, 576, 147-168.

[18] Kantam, M. L.; Roy, M.; Roy, S.; Sreedhar, B.; Madhavendra, S. S.; Choudary, B. M.; De, R. L. Polyaniline supported palladium catalyzed Suzuki-Miyaura cross-coupling of bromo- and chloroarenes in water. Tetrahedron 2007, 63, 8002-8009.

[19] Lemo, J.; Heuze, K.; Astruc, D. Efficient and recyclable dendritic Buchwald-type catalyst for the Suzuki reaction. Chem. Commun. 2007, 4351-4353.

[20] Ishiyama, T.; Kizaki, H.; Hayashi, T.; Suzuki, A.; Miyaura, N. Palladium-catalyzed carbonylative cross-coupling reaction of arylboronic acids with aryl electrophiles: Synthesis of biaryl ketones. J. Org. Chem. 1998, 63, 4726-4731.

[21] Corma, A.; Garcia, H.; Leyva, A. Comparison between polyethylenglycol and imidazolium ionic liquids as solvents for developing a homogeneous and reusable palladium catalytic system for the Suzuki and Sonogashira coupling. Tetrahedron 2005, 61, 9848-9854.

[22] Fan, X. B.; Peng, W. C.; Li, Y.; Li, X. Y.; Wang, S. L.; Zhang, G. L.; Zhang, F. B. Deoxygenation of exfoliated graphite oxide under alkaline conditions: A green route to graphene preparation. Adv. Mater. 2008, 20, 4490-4493.

[23] Lerf, A.; He, H. Y.; Forster, M.; Klinowski, J. Structure of graphite oxide revisited. J. Phys. Chem. B 1998, 102, 4477-4482.

[24] Karousis, N.; Tsotsou, G. E.; Evangelista, F.; Rudolf, P.; Ragoussis, N.; Tagmatarchis, N. Carbon nanotubes decorated with palladium nanoparticles: Synthesis, characterization, and catalytic activity. J. Phys. Chem. C 2008, 112, 13463-13469.

[25] Lu, J. S. Effect of surface modifications on the decoration of multi-walled carbon nanotubes with ruthenium nanoparticles. Carbon 2007, 45, 1599-1605.

[26] Wang, H.; Bash, R.; Lindsay, S. M.; Lohr, D. Solution AFM studies of human Swi-Snf and its interactions with MMTV DNA and chromatin. Biophys. J. 2005, 89, 3386-3398.

[27] Nemec, N.; Tomanek, D.; Cuniberti, G. Contact dependence of carrier injection in carbon nanotubes: An ab initio study. Phys. Rev. Lett. 2006, 96, 076802.

[28] Yang, D. X.; Velamakanni, A.; Bozoklu, G.; Park, S. J.; Stoller, M.; Piner, R. D.; Stankovich, S.; Jung, I.; Field, D. A.; Ventrice, C. A.; Ruoff, R. S. Chemical analysis of graphene oxide films after heat and chemical treatments by X-ray photoelectron and micro-Raman spectroscopy. Carbon 2009, 47, 145-152.

[29] Jasuja, K.; Berry, V. Implantation and growth of dendritic gold nanostructures on graphene derivatives: Electrical property tailoring and Raman enhancement. ACS Nano 2009, 3, 2358-2366.

[30] Lin, Y.; Watson, K. A.; Fallbach, M. J.; Ghose, S.; Smith, J. G.; Delozier, D. M.; Cao, W.; Crooks, R. E.; Connell, J. W. Rapid, solventless, bulk preparation of metal nanoparticledecorated carbon nanotubes. ACS Nano 2009, 3, 871-884. 
[31] Tong, L. M.; Li, Z. P.; Zhu, T.; Xu, H. X.; Liu, Z. F. Single gold-nanoparticle-enhanced Raman scattering of individual single-walled carbon nanotubes via atomic force microscope manipulation. J. Phys. Chem. C 2008, 112, 7119-7123.

[32] Krasheninnikov, A. V.; Lehtinen, P. O.; Foster, A. S.; Pyykkö, P.; Nieminen, R. M. Embedding transition-metal atoms in graphene: Structure, bonding, and magnetism. Phys. Rev. Lett. 2009, 102, 126807.

[33] Okamoto, Y. Density-functional calculations of icosahedral $\mathrm{M}_{13}(\mathrm{M}=\mathrm{Pt}$ and $\mathrm{Au})$ clusters on graphene sheets and flakes. Chem. Phys. Lett. 2006, 420, 382-386.

[34] Kudin, K. N.; Ozbas, B.; Schniepp, H. C.; Prud'homme, R. K.; Aksay, I. A.; Car, R. Raman spectra of graphite oxide and functionalized graphene sheets. Nano Lett. 2008, 8, 36-41.

[35] Zeng, H. B.; Cai, W. P.; Li, Y.; Hu, J. L.; Liu, P. S. Composition/structural evolution and optical properties of $\mathrm{ZnO} / \mathrm{Zn}$ nanoparticles by laser ablation in liquid media. $J$. Phys. Chem. B 2005, 109, 18260-18266.

[36] Mafune, F.; Kohno, J.; Takeda, Y.; Kondow, T. Formation and size control of silver nanoparticles by laser ablation in aqueous solution. J. Phys. Chem. B 2000, 104, 9111-9117.

[37] Santra, A. K.; Goodman, D. W. Oxide-supported metal clusters: Models for heterogeneous catalysts. J. Phys.-Condens. Mat. 2003, 15, R31-R62.

[38] Turner, M.; Golovko, V. B.; Vaughan, O. P. H.; Abdulkin, P.; Berenguer-Murcia, A.; Tikhov, M. S.; Johnson, B. F. G.; Lambert, R. M. Selective oxidation with dioxygen by gold nanoparticle catalysts derived from 55-atom clusters. Nature 2008, 454, 981-983.

[39] Zapf, A.; Ehrentraut, A.; Beller, M. A new highly efficient catalyst system for the coupling of nonactivated and deactivated aryl chlorides with arylboronic acids. Angew.
Chem. Int. Edit. 2000, 39, 4153-4155.

[40] Littke, A. F.; Dai, C. Y.; Fu, G. C. Versatile catalysts for the Suzuki cross-coupling of arylboronic acids with aryl and vinyl halides and triflates under mild conditions. J. Am. Chem. Soc. 2000, 122, 4020-4028.

[41] Goossen, L. J.; Ghosh, K. Palladium-catalyzed synthesis of aryl ketones from boronic acids and carboxylic acids or anhydrides. Angew. Chem. Int. Edit. 2001, 40, 3458-3460.

[42] Xin, B. W.; Zhang, Y. H. Cheng, K. The surfactant-promoted cross-coupling reactions of arylboronic acids with carboxylic anhydrides or acyl chlorides in water. Synthesis-Stuttgart 2007, 1970-1978.

[43] Harada, T.; Ikeda, S.; Miyazaki, M.; Sakata, T.; Mori, H.; Matsumura, M. A simple method for preparing highly active palladium catalysts loaded on various carbon supports for liquid-phase oxidation and hydrogenation reactions. $J$. Mol. Catal. A-Chem. 2007, 268, 59-64.

[44] Diallo, A. K.; Ornelas, C.; Salmon, L.; Aranzaes, J. R.; Astruc, D. "Homeopathic" catalytic activity and atom-leaching mechanism in Miyaura-Suzuki reactions under ambient conditions with precise dendrimer-stabilized Pd nanoparticles. Angew. Chem. Int. Edit. 2007, 46, 8644-8648.

[45] Corma, A.; Garcia, H.; Leyva, A. Catalytic activity of palladium supported on single wall carbon nanotubes compared to palladium supported on activated carbon. Study of the Heck and Suzuki couplings, aerobic alcohol oxidation and selective hydrogenation. J. Mol. Catal. A-Chem. 2005, 230, 97-105.

[46] Alonso, F.; Beletskaya, I. P.; Yus, M. Non-conventional methodologies for transition-metal catalysed carbon-carbon coupling: A critical overview. Part 2: The Suzuki reaction. Tetrahedron 2008, 64, 3047-3101. 